\title{
Turbulent fluid flow over aerodynamically rough surfaces using direct numerical simulations
}

\author{
M. Thakkar, A. Busse, and N.D. Sandham
}

\section{Abstract}

Incompressible turbulent fluid flow in aerodynamically rough channels is investigated using direct numerical simulations. A comprehensive database of simulation data for rough surfaces with different topographical properties has been developed for 17 industrially relevant rough surface samples. It includes numerous commonlyseen industrial rough surfaces such as concrete, graphite, carbon-carbon composite and ground, shotblasted and spark-eroded steel. Other surfaces such as cast, filed and gritblasted steel are also studied, along with replicas of ship propeller surfaces eroded by periods of service. The Reynolds number considered is $R e_{\tau}=180$, for which the flow is in the transitionally rough regime. A study with variable $\delta / S_{q}$ ratio while keeping $S_{q}^{+}$constant, where $S_{q}$ is the root mean squared roughness height, is conducted for one of the samples with the mean profiles showing convergence for $\delta / S_{q}>\approx 25$. A Reynolds number dependence study is conducted for two of the samples with $R e_{\tau}$ up to 720 showing a more complete range up to the fully rough flow regime, allowing the equivalent sandgrain roughness height, $k_{s}$ to be computed. A correlation based on the frontal and wetted roughness area is found to be superior to the surface skewness in predicting $\Delta U^{+}$based on the topographic surface parameters.

\footnotetext{
M. Thakkar · N.D. Sandham

University of Southampton, Southampton, UK. e-mail: \{mnt2g11, n. sandham\}@soton . ac.uk
}

A. Busse

University of Glasgow, Glasgow, UK. e-mail: angela.busse@glasgow.ac.uk 


\section{Introduction}

In many boundary layer flows (especially geophysical flows), surface roughness is not the exception but the norm. A wide range of practical applications show the presence of roughness at various scales, including industrial finishing processes such as grinding and shotblasting [2], industrial heat exchangers, ship propellers and hulls due to marine fouling, plant canopies on the earth's surface and urban flows over cities. The primary effect of roughness is an increase in the surface friction compared to a smooth wall, which is seen as a downward shift in the mean streamwise velocity profile when plotted in wall units. This shift is quantified by the roughness function, $\Delta U^{+}$. Roughness also causes an increase in the exchange of momentum in the near-wall regions and in the wall heat and mass transfer rates.

Most previous experimental and numerical studies of flows over rough walls have dealt with geometries that were artificially constructed from simple roughness elements e.g. bars, cubes and spheres, arranged in regular patterns. Such surfaces typically possess a small number of characteristic length scales and their surface properties such as roughness heights and spacing can be easily evaluated. They are simple to construct for the purpose of experimentation or computational simulations. However, most rough surfaces encountered in practice are irregular in nature and have features on a large number of length scales and a complex topography. They bear limited resemblance to the above-mentioned regular rough surfaces and it is not guaranteed that ideas developed from studies over regular rough surfaces can be applied to irregular rough surfaces.

The overall aim of this research is to understand the fluid flow characteristics over irregular and more realistic roughness, as opposed to the regular and idealised rough surface geometries studied extensively in the past. This is done by studying incompressible turbulent flow through rough channels using direct numerical simulations (DNS).

\section{Methodology}

A three step methodology [1] is used to conduct the rough wall channel flow simulations. In the first step, surface data is acquired from the physical sample by using a variable focus microscope. The second step involves data pre-processing where the raw data is filtered in Fourier space in order to eliminate any measurement noise. Filtering also removes the smallest roughness length scales (which can be regarded as aerodynamically irrelevant [6]) and provides a periodic tile required for the DNS. The final step involves using the periodic tile as a no-slip boundary condition in the DNS of turbulent channel flow. The study is conducted at $R e_{\tau}=u_{\tau} \delta / v=180$, where $u_{\tau}$ is the friction velocity, $\delta$ is the channel half-height and $v$ is the kinematic viscosity. The rough surface is resolved using an immersed boundary method that is an iterative modification of [9]; details of the method are given in [1]. The mean surface height is set as the mean reference plane, $z=0$ (where $z$ is the wall- 
normal direction). The distance between the bottom and top mean reference planes is $2 \delta$. Uniform grid spacing is used in $x$ and $y$ taking the minimum of two criteria: (a) $\Delta x^{+}=\Delta y^{+} \approx 5$ and (b) $\Delta x=\Delta y \approx \lambda_{\min } / 12$ where $\lambda_{\text {min }}$ is the smallest wavelength of the rough surface after filtering. A stretched grid is used in the wallnormal direction with $\Delta z_{\min }^{+}<1$ close to the rough walls and $\Delta z_{\max }^{+} \leq 5$ toward the channel centre. All rough surfaces are scaled to the same physical roughness height, defined by the mean-peak-to-valley height, $S_{z, 5 \times 5}{ }^{1}$, such that $\delta / S_{z, 5 \times 5}=6$ (and $20<\delta / S_{q}<31$ ). The roughness height, $k$ is defined by $S_{z, 5 \times 5}$ unless stated otherwise.

\section{Results}

The mean profiles for $R e_{\tau}=180$ for all surfaces are shown in figures 1 and 2. Due to the large number of surfaces, these results have been spread out over two plots; figure 1 shows profiles from previous studies [2] and figure 2 shows additional profiles from the present study. All surfaces have a clear effect on the mean flow as can be seen from the downward shift, $\Delta U^{+}$in the mean velocity profile, compared to a smooth wall. $\Delta U^{+}$is generally measured as the vertical shift of the log region of the mean velocity profile but since the Reynolds number in this study is low, no clearly defined log region is present. Hence $\Delta U^{+}$is obtained by subtracting the centreline velocity for a given sample from the corresponding smooth-wall centreline velocity [3]. Table 1 displays the $\Delta U^{+}$values for all surfaces. There is a wide range, from 1.3 to 5 , despite all the surfaces being scaled to the same roughness height. This is a clear indication that the roughness function depends not only on the roughness height for a given surface but also on the detailed roughness topography.

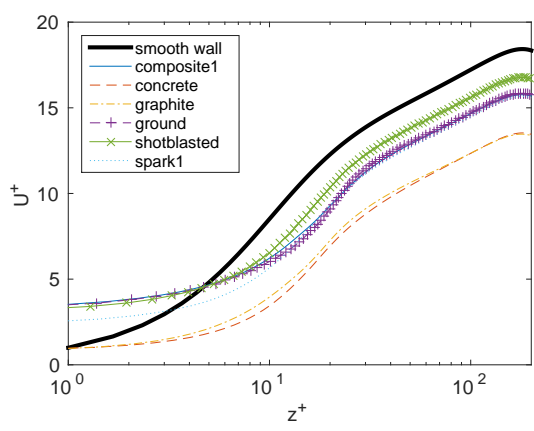

Fig. 1 mean profiles for $\operatorname{Re}_{\tau}=180(6$ surfaces)

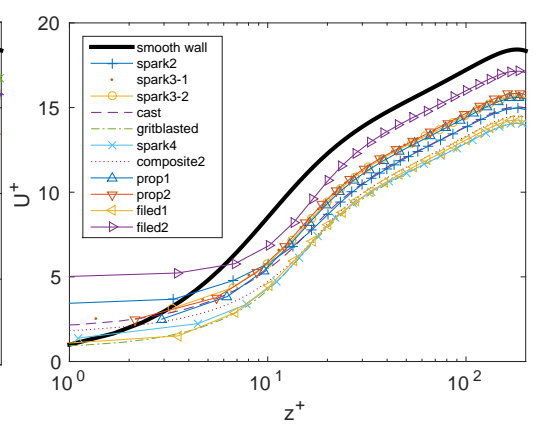

Fig. 2 mean profiles for $R e_{\tau}=180(11$ surfaces)

\footnotetext{
${ }^{1}$ To compute $S_{z, 5 \times 5}$, a surface is divided into $5 \times 5$ sections of equal size. For each section, the maximum and minimum surface height is found. The mean-peak-to-valley height is the difference between the mean of the maxima and the mean of the minima.
} 
Table $1 \Delta U^{+}$for all samples

\begin{tabular}{cccccccccc}
\hline & composite1 & concrete & graphite & ground & shotblasted spark1 & spark2 & spark3-1 & spark3-2 \\
\hline$\Delta U^{+}$ & 2.7 & 4.9 & 5.0 & 2.6 & 1.7 & 2.8 & 3.5 & 2.7 & 2.8 \\
\hline & cast & gritblasted & spark4 & composite2 & prop1 & prop2 & filed1 & filed2 & \\
\hline$\Delta U^{+}$ & 3.4 & 4.4 & 4.4 & 3.9 & 2.8 & 2.6 & 4.2 & 1.3 & \\
\hline
\end{tabular}

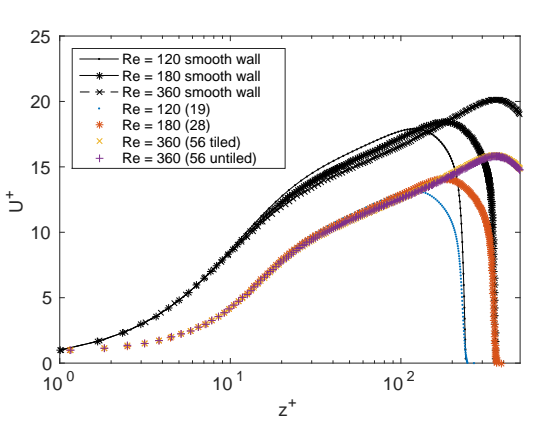

Fig. 3 Mean profiles for $\delta / S_{q}$ study (gritblasted surface). Numbers in brackets in the legend indicate $\delta / S_{q}$ values

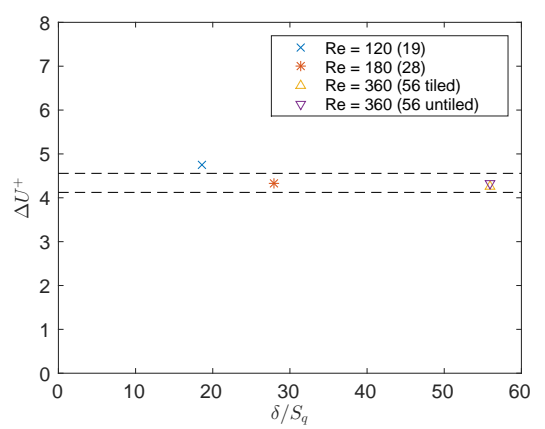

Fig. $4 \Delta U^{+}$against $\delta / S_{q}$ (gritblasted surface). Numbers in brackets in the legend indicate $\delta / S_{q}$ values

It is commonly assumed that universal behaviour emerges only when the roughness height is considerably smaller than the macroscopic length scale of the flow, i.e. for high $\delta / k$ [5]. In order to check the sensitivity of the results to $\delta / k$, a study is conducted for the gritblasted sample. Here the roughness height is chosen as the root mean squared roughness height, $S_{q}$. The ratio, $\delta / S_{q}$ is varied but the roughness height Reynolds number, $S_{q}^{+}=S_{q} u_{\tau} / v$ is kept constant for all cases. For instance, $S_{q}^{+} \approx 6.44$ at $R e_{\tau}=180$, for which $\delta / S_{q} \approx 28$. Other cases include $R e_{\tau}=120,360$ with corresponding $\delta / S_{q} \approx 19,56$ respectively, obtained by scaling the $\operatorname{Re}_{\tau}=180$ case. Due to the small roughness height in the $R e_{\tau}=360$ case the domain size may be too small for proper flow development. Hence this sample was replicated in $x$ and $y$ to give a tiled sample with a larger domain size. Both cases were simulated. The need for this replication motivated the choice of $S_{q}$ instead of $S_{z, 5 \times 5}$ for the roughness height as the latter does not scale by exactly the same factor as the irregular surface. As seen from figure 3, the mean profiles for all cases are similar. It can also be seen from figure 4, which shows $\Delta U^{+}$against $\delta / S_{q}$, that convergence for $\Delta U^{+}$ is obtained for $\delta / S_{q}>\approx 25$. The dashed lines in this figure represent $5 \%$ tolerance bands around the $R e_{\tau}=180$ case.

Figure 5 shows the dependence of $\Delta U^{+}$on $R e_{\tau}$ for two surfaces, graphite and gritblasted. It also shows Colebrook's universal interpolation formula [5] given by $\Delta U^{+}\left(k_{s}^{+}\right)=\kappa^{-1} \log \left(0.3 k_{s}^{+}+1\right)$ and the fully rough asymptote line given by $\Delta U^{+}\left(k_{s}^{+}\right)=\kappa^{-1} \log \left(0.3 k_{s}^{+}\right)$, where $k_{s}$ is the equivalent sand grain roughness height and $\kappa \approx 0.41$ is the von Kármán constant. The range of $R e_{\tau}=90,120,180,240,360$, 


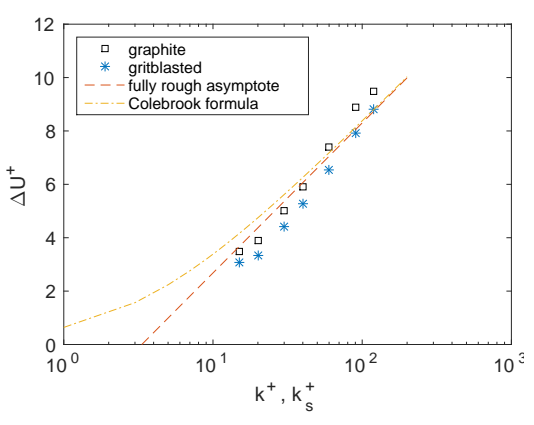

Fig. $5 R e_{\tau}$ dependence study

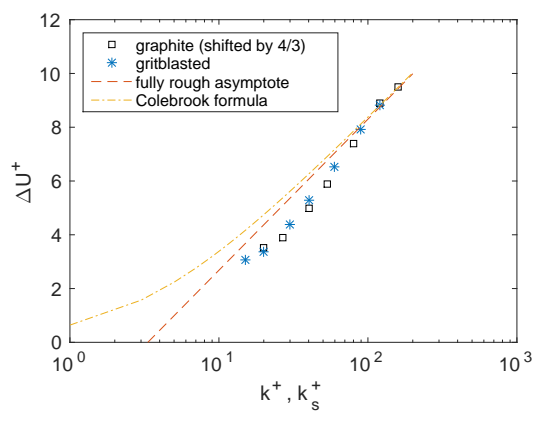

Fig. $6 R e_{\tau}$ dependence study. Graphite sample results shifted horizontally by applying a factor of $4 / 3$

540,720 gives corresponding $k^{+}=15,20,30,40,60,90,120$ (with $\delta / k=6$ for all $R e_{\tau}$ ) respectively. This curve is expected to asymptote to a straight line at high $k^{+}$ corresponding to the fully rough regime, and allowing the definition of an equivalent sand grain roughness height, $k_{s}^{+}$, as was also noted by Yuan \& Piomelli [10]. Thus in figure 6 , points for graphite have been shifted horizontally by $4 / 3$ such that points in the fully rough regime fall on the asymptote. This gives $k_{s}^{+} \approx(4 / 3) k^{+}$for the graphite surface and $k_{s}^{+} \approx k^{+}$for the gritblasted surface. For $k^{+}<\approx 30$, the points depart from the expected trend probably due to low Reynolds number effects.

A number of numerical models that relate $\Delta U^{+}$to the surface topographical properties of the three-dimensional rough surfaces have been proposed in the past [4]. The generalised Sigal-Danberg parameter, $\Lambda_{s}$ [7] as modified by van Rij et al [8] is a geometrical parameter that captures information about the roughness shape as well as direction with respect to the mean flow. It is defined as $\Lambda_{s}=\left(S / S_{f}\right)\left(S_{f} / S_{s}\right)^{-1.6}$, where $S$ is the planform area of the corresponding smooth

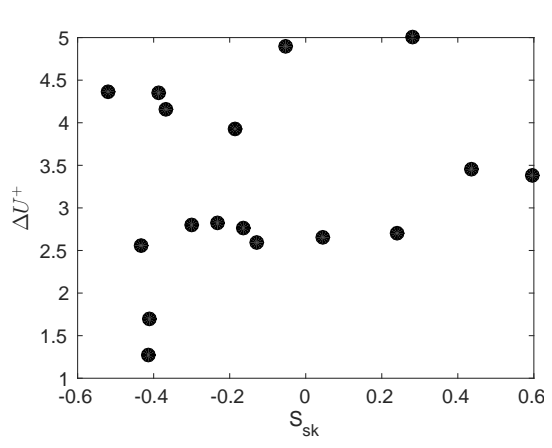

Fig. $7 \Delta U^{+}$against surface skewness, $S_{s k}$. Symbols indicate different rough surfaces

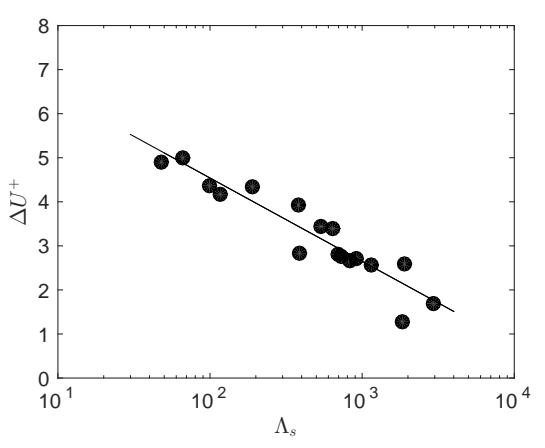

Fig. $8 \Delta U^{+}$against Sigal-Danberg parameter, $\Lambda_{s}$. Symbols indicate different rough surfaces 
surface, $S_{f}$ is the total projected frontal area of all the roughness elements and $S_{S}$ is the total area of all roughness elements wetted by the flow. $S / S_{f}$ is a roughness density parameter and $S_{f} / S_{s}$ is a roughness shape parameter. As shown in figures 7 and 8, surface skewness, $S_{s k}$ fails to correlate the current data whereas $\Delta U^{+}$decreases with increasing $\Lambda_{s}$. The best fit is given by $\Delta U^{+}=\log \left(\Lambda_{s}\right)^{-1.89}+8.32$.

\section{Conclusions}

Incompressible turbulent channel flow over a large variety of industrially-relevant rough surfaces has been studied using direct numerical simulations. Mean velocity profiles for the surfaces show significant variation in terms of $\Delta U^{+}$, despite all surfaces being scaled to the same physical roughness height, clearly indicating that the roughness effect depends on the detailed surface topography as well as the roughness height. A sensitivity study to $\delta / S_{q}$ while keeping $S_{q}^{+}$constant for one sample showed that similar mean profiles are obtained for the cases considered and convergence for $\Delta U^{+}$is obtained for $\delta / S_{q}>\approx 25$. Simulations over a wide range of $R e_{\tau}$ from the transitionally rough regime up to the fully rough regime for two of the samples allowed the calculation of an equivalent sand grain roughness height, $k_{s}$. The Sigal-Danberg parameter, $\Lambda_{s}$ was found to correlate much better with $\Delta U^{+}$ than the surface skewness, $S_{s k}$.

\section{References}

1. Busse, A., Lützner, M. \& Sandham, N.D. Direct numerical simulation of turbulent flow over a rough surface based on a surface scan. Computers \& Fluids. 116: 129-147 (2015)

2. Busse, A., Tyson, C.J., Sandham, N.D. \& Lützner, M. DNS of Turbulent channel flow over engineering rough surfaces. In: Eight International Symposium on Turbulence and Shear Flow Phenomena (TSFP-8), August 28-30 2013, Poitiers, France

3. Busse, A. \& Sandham, N.D. Parametric forcing approach to rough-wall turbulent channel flow. J. Fluid Mech. 712: 169-202 (2012)

4. Flack, K.A. \& Schultz, M.P. Review of hydraulic roughness scales in the fully rough regime. J. Fluids Eng. 132: 041203-1-041203-10 (2010)

5. Jiménez, J. Turbulent flows over rough walls. Annu. Rev. Fluid Mech. 36: 173-196 (2004)

6. Mejia-Alvarez, R. \& Christensen, K.T. Low-order representations of irregular surface roughness and their impact on a turbulent boundary layer. Phys. Fluids. 22: 015106 (2010)

7. Sigal, A. \& Danberg, J.E. Analysis of turbulent boundary-layer over rough surfaces with application to projectile aerodynamics. Technical Report BRL-TR-2977. Army Ballistic Research Lab, Aberdeen Proving Grounds, MD (1988)

8. van Rij, J.A., Belnap, B.J. \& Ligrani, P.M. Analysis and experiments on three-dimensional irregular surface roughness. J. Fluids Eng. 124(3): 671-677 (2002)

9. Yang, J. \& Balaras, E. An embedded-boundary formulation for large-eddy simulation of turbulent flows interacting with moving boundaries. J. Comput. Phys. 215: 12-40 (2006)

10. Yuan, J. \& Piomelli, U. Estimation and prediction of the roughness function on realistic surfaces. Journal of Turbulence. 15(6): 350-365 (2014) 